\title{
Five Maturity Levels of Managing AI: From Isolated Ignorance to Integrated Intelligence
}

\author{
Ulrich Lichtenthaler \\ lichtenthaler@web.de | ISM - International School of Management, Im MediaPark 5c, 50670 Cologne, \\ Germany
}

\begin{abstract}
To guide future discussions about managing artificial intelligence (AI), this article suggests an AI management framework with five maturity levels, which are comparable to the five levels of the autonomous driving framework from no automation to complete automation. If companies move beyond Isolated Ignorance (Level 0), they are characterized by an Initial Intent (Level 1), which typically evolves towards an Independent Initiative (Level 2). A more advanced management leads to Interactive Implementation (Level 3) and Interdependent Innovation (Level 4). On this basis, a close combination of AI and human knowledge enables a sustainable competitive advantage with Integrated Intelligence (Level 5). This framework draws on the intelligence-based approach to company performance, and it provides the basis for an AI maturity assessment in organizations. It further helps to identify many firms' managerial challenges as well as major organizational limitations even in those firms that are often considered as AI leaders.
\end{abstract}

Keywords. Artificial Intelligence; Conceptual Framework; Digitalization; Digital Transformation; Integrated Intelligence; Intelligence-based View.

Cite paper as: Lichtenthaler, U., (2020). Five Maturity Levels of Managing AI: From Isolated Ignorance to Integrated Intelligence, Journal of Innovation Management, www.open-jim.org, 8(1), 39-50. 


\section{Introduction}

"If you invent a breakthrough in artificial intelligence, so machines can learn, that is worth 10 Microsofts" (Lohr, 2004). Bill Gates made this statement already in 2004, and the evolution of artificial intelligence (AI) has progressed substantially since then (Daugherty \& Wilson, 2018; Davenport, 2018). Beyond the firms that focus on developing and commercializing new AI technology, many established manufacturing and service companies have started strategic initiatives to apply AI solutions, such as advanced analytics and smart algorithms. This massive attention to AI may be observed in nearly all sectors, including consumer products, machinery, automotive, financial services, electronics, and many others (Agrawal, Gans, \& Goldfarb, 2018; Apanesevic, Arvidsson, \& Markendahl, 2018; Brock \& Wangenheim, 2019). Despite the broad application of AI, the knowledge about managing such initiatives is surprisingly limited. This is particularly noteworthy because many firms' AI initiatives do not live up to the initial expectations, whereas only a few firms and organizations achieve major competitive benefits from leveraging the recent progress in different AI fields, such as natural language processing, machine learning, and speech synthesis (Brito, 2018; Haenlein \& Kaplan, 2019; Tambe, Cappelli, \& Yabukovich, 2019).

While some firms still ignore AI, many others pursue strategic AI programs, but the management of these programs has often remained a black box, and our knowledge about managing AI has remained incomplete so far beyond some initial insights (Garbuio \& Lin, 2019; Lichtenthaler, 2020b; Shrestha, Ben-Menahem, \& Krogh, 2019). This insufficient understanding of managing AI is similar to the limited insights into the results of some machine learning algorithms based on big data (Plastino \& Purdy, 2018; Rometty, 2016). To guide a deeper understanding of AI management, this article builds on prior research in the Journal of Innovation Management about the intelligence-based view (Lichtenthaler, 2019) and suggests a framework with five maturity levels of managing AI. These five levels are comparable to the five well-known levels of autonomous driving from no automation to complete automation (SAE International, 2019).

This AI management framework offers several contributions. First, it provides the basis for an AI maturity assessment in companies, and it illustrates the relevance of an intelligence-based view of firm performance (Kumar, Rajan, Venkatesan, \& Lecinski, 2019; Lichtenthaler, 2019). Second, the different maturity levels help to explain many companies' challenges in managing AI initiatives and in profiting from these initiatives by strengthening their competitive position (Datta, 2018; Overgoor, Chica, Rand, \& Weishampel, 2019). Third, the framework allows for identifying the limitations of many firms that are considered to be successfully managing AI (Metcalf, Askay, \& Rosenberg, 2019; Swift, 2018). These companies are often in the paradoxical situation that they only believe to leverage AI, but a more detailed analysis points to many unrealized opportunities that could be achieved with an integrated intelligence architecture (Bühring \& Moore, 2018; Wilbert, Durst, Ferenhof, \& Selig, 2018).

\section{Managing artificial intelligence}

Many previous articles and studies about AI focus on specific topics, and this varying emphasis has contributed to a relatively broad understanding of what AI actually is, for example with 
regard to advanced data analytics and smart algorithms (Lichtenthaler, 2020b). In particular, some previous studies have focused on technological opportunities and solutions, whereas others have primarily concentrated on market applications and use cases of AI (Davenport, 2018; Tegmark, 2018). In addition, the understanding of AI is often dynamic, which leads to a different scope of AI as technologies and markets evolve over time (Lichtenthaler, 2020b). In the management context, AI may be defined as "a system's ability to interpret external data correctly, to learn from such data, and to use those learnings to achieve specific goals and tasks through flexible adaptation" (Kaplan \& Haenlein, 2019, p. 15).

In a similar vein, the glossary of the research and advisory firm Gartner provides the following definition of AI. "Artificial intelligence (AI) applies advanced analysis and logic-based techniques, including machine learning, to interpret events, support and automate decisions, and take actions" (Gartner, 2019a). On this basis, there are various systematizations of specific fields of AI, and some of these categorizations focus on different fields of technology, whereas others describe distinct market aspects (Lichtenthaler, 2020b). In this regard, machine learning has probably been discussed most prominently in recent years in light of the substantial technological improvements in this field (Finlay, 2017). Other important types of AI include natural language processing, speech synthesis, machines and robotics, expert systems, computer vision as well as optimization and planning solutions. Different overviews may be found in several prior works (Haenlein \& Kaplan, 2019; Lichtenthaler, 2020b; Mueller \& Massaron, 2018; Wein, 2018).

On this basis, the management of AI rather than AI generally comprises the following activities, and this understanding of AI management as the coordination of a firm's AI activities follows the understanding of data management by the research and advisory firm Gartner, which focuses on the coordination of data in an enterprise (Gartner, 2019b). Consistent with this underlying logic, AI management consists of the strategies, processes, practices, activities, tools, and organizational mechanisms for achieving consistent access and utilization of artificial intelligence in response to the intelligence requirements across an organization. As such, the AI activities are part of a company's intelligence architecture, which comprises various types of AI, various types of human intelligence, and a meta-intelligence for renewing and recombining the intelligence types and their interfaces (Lichtenthaler, 2020b). This understanding of intelligence architecture draws on the concept of Integrated Intelligence and the intelligence-based view of company performance (Lichtenthaler, 2019, 2020b).

In particular, this notion of an intelligence architecture is consistent with the concept of information architecture in information technology management. In this regard, the research and advisory firm Gartner defines information architecture as follows: "All the sources of information - including paper, graphics, video, speech and thought - that define the enterprise are represented by this layer of applications architecture. It also defines the sources and destinations of information, its flow through the enterprise, as well as the rules for persistence, security and ownership" (Gartner, 2019d). As such, the enterprise information architecture "describes through a set of requirements, principles and models - the current state, future state and guidance necessary to flexibly share and exchange information assets to achieve effective enterprise change" (Gartner, 2019c). In this respect, an integrated intelligence architecture involves a comprehensive perspective on the different types of AI, the different types of human intelligence and 
the meta-intelligence to ensure that the overall architecture is more the sum of the individual intelligence types (Lichtenthaler, 2020b).

\section{Five maturity levels}

Based on the detailed coverage of the recent progress in AI across all media channels, most companies are well aware of the growing competitive relevance of AI (Agrawal, Gans, \& Goldfarb, 2017; Plastino \& Purdy, 2018). Nonetheless, many businesses still largely ignore the increasing importance of AI. In some firms, this reluctant approach derives from a limited openness to new technology and a limited willingness to innovate (Huang \& Rust, 2018; Ili \& Lichtenthaler, 2017). In contrast, some other companies have taken the deliberate strategic decision to focus on other value drivers for their customers. Consequently, they may take major steps to apply AI solutions in the future. Currently, however, they continue to concentrate on other topics and are largely inactive with respect to AI (Kavadias, Ladas, \& Loch, 2016; Tegmark, 2018).

Therefore, this reluctant approach has been termed Isolated Ignorance (Lichtenthaler, 2020b), and it may be considered Level 0 in managing AI based on an intelligence-based perspective on firm performance. If companies leave this stage of inactivity and start some initial steps of experimenting with selected AI solutions, they enter Level 1 of managing AI. This level of Initial Intent is often characterized by exploring the technical feasibility as well as the business viability of specific AI applications (Figure 1). However, many of these experimental activities are discontinued after some time, and they are not rolled out in the organization because of major uncertainties whether the implementation of these particular AI solutions will actually pay off or not in the long run.

Level 2 of managing AI is termed Independent Initiative. Here, companies typically have several ongoing AI activities, whose extent is still relatively limited. In large businesses, these activities have often been started in various organizational units, and there is hardly any coordination among these activities. For example, large insurance companies like American International Group and Zurich started multiple AI programs in different business and functional units several years ago (Lloyd-Jones, 2016). Usually, these AI programs focus on advanced automation solutions to improve the efficiency of established business processes by automating jobs (Agrawal et al., 2018). If companies start to exploit diverse business opportunities deriving from AI, they enter Level 3, which is called Interactive Implementation. These companies pursue multiple AI solutions, whose implementation is aligned with one another. On this basis, the executives coordinate the activities across multiple units of large organizations. In addition, there may be pooled interdependencies between AI and human intelligence, which provide distinct contributions to a firm's overall intelligence architecture (Lichtenthaler, 2018).

Many companies that are regarded as AI leaders have achieved Level 3. However, many of these firms still focus on utilizing AI for optimization purposes rather than for developing novel solutions for their existing and new customers (Lichtenthaler, 2020b). This is the core difference between those firms and the small minority of businesses that have arrived at Level 4 of Interdependent Innovation. These companies put a much stronger emphasis on growth and innovation due to combining AI solutions with additional innovation types, for example new services and 


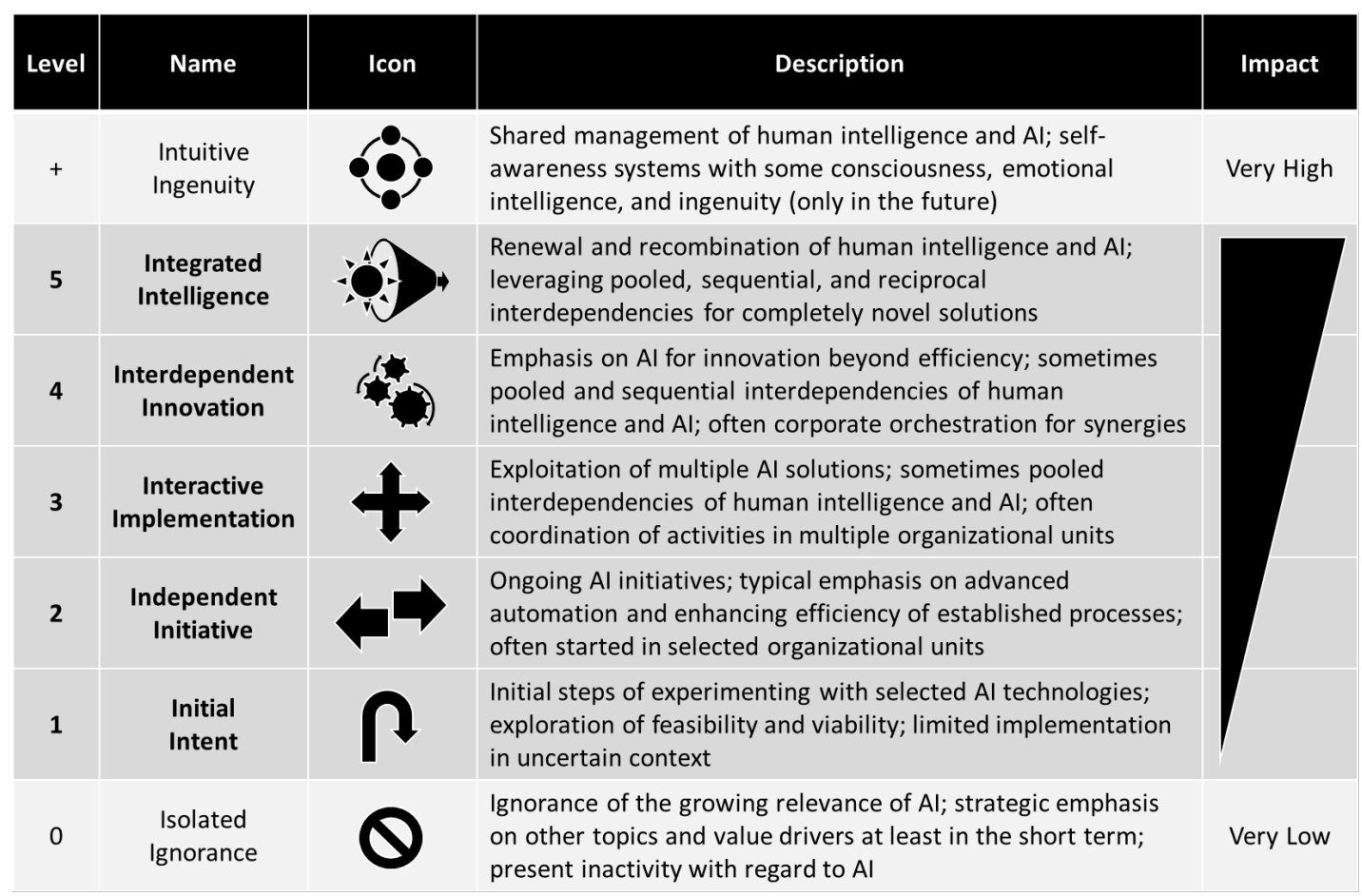

Fig. 1. AI management framework with five maturity levels.

new business models (Davenport, 2018). In addition to pooled interdependencies between AI and human intelligence, there may be sequential interdependencies in situations when the output of one intelligence constitutes the input to the other intelligence (Lichtenthaler, 2018). For instance, Autodesk's AI solution Dreamcatcher enables designers to ease the identification of suitable solutions based on particular features that are considered as fixed (Wilson \& Daugherty, 2018; Wilson, Daugherty, \& Bianzino, 2017). This approach facilitates the design process, and it involves close interactions with multiple other experts and solutions in distinct organizational units. Accordingly, Level 4 also calls for a systematic orchestration of AI programs and additional initiatives to achieve synergies and strategic alignment. For example, General Electric specifically regards its AI activities as a multidisciplinary program, which considers efficiency as well as innovation benefits, including advanced analytics and edge computing for strengthening its industrial IoT solutions (General Electric, 2019).

Finally, Level 5 refers to Integrated Intelligence. To date, hardly any company has arrived at this level of managing AI, which would involve a meta-intelligence for systematically renewing and recombining AI and human intelligence (Lichtenthaler, 2020a). Thus, companies at this level fully go beyond viewing AI applications as stand-alone solutions. Instead, firms try to achieve an integrated intelligence architecture by leveraging all types of interdependencies between AI and human intelligence - pooled, sequential, and reciprocal interdependencies (Lichtenthaler, 2018). In the case of reciprocal interdependencies, there are cyclical interactions between AI and human intelligence (Lichtenthaler, 2018). Based on an integrated intelligence architecture, companies may arrive at completely novel solutions along a development funnel with close collaborations of 
AI and human experts. For example, the startup Tawny.ai develops tailored algorithms drawing on its key technology for AI-powered emotion analytics (Lichtenthaler, 2020b). On this basis, it aims at easing the interaction between $\mathrm{AI}$ and human intelligence to enable integrated intelligence architectures on a scalable basis.

Even if some businesses are considered AI leaders - or if they consider themselves as AI leaders - they usually have not achieved Level 5 , at least not in large parts of their organizations. Consequently, they still do not leverage the full strategy space of AI. Many of these companies are already very successful in terms of optimizing and streamlining their internal processes to achieve cost reductions (Agrawal et al., 2018; Daugherty \& Wilson, 2018). However, the executives typically do not consider the variety of effects that AI will have on the value chain in their industry. In addition, the opportunities for business model innovations, potentially together with external partners in a future intelligence-based ecosystem, are often left unconsidered. Thus, even the most advanced companies in managing AI are only able to gain an intelligence-based competitive advantage (Lichtenthaler, 2020b). However, they will have a difficult time in sustaining this competitive advantage over a longer period, especially as many AI applications will become relatively standardized solutions in the near future, which are further commoditized in the long run. Figure 1 indicates that the competitive consequences of AI are strong in the most advanced companies because they have often achieved Level 4 of managing AI. Nonetheless, there is still significant unrealized potential for further strengthening their competitive positions based on an integrated intelligence architecture.

Level 5 of managing AI constitutes the top level that companies may achieve at present. At some point in the future, however, there may be an additional level which goes beyond today's maximum (Finlay, 2017; Mueller \& Massaron, 2018). In the context of further substantial evolutions of various technology fields, management may be further shared among AI and human executives. Consequently, the competitive impact of AI would be even higher, and this Level + would refer to Intuitive Ingenuity. This additional level would require self-awareness systems with some degree of consciousness, which constitute the form of AI that is envisioned in some science fiction movies (Mueller \& Massaron, 2018). On this basis, AI solutions would comprise some degree of emotional intelligence, ingenuity, and intuition. These solutions may be able to accomplish a larger portion of the activities that currently still require uniquely human skills, such as creativity, motivation, and storytelling (Lichtenthaler, 2018). Throughout the next decades, nearly all companies will move along the different maturity levels from Level 0 to Level 5 with a growing relevance of Integrated Intelligence - and potentially even beyond this level due to future scientific evolutions in AI and similar scientific fields.

\section{Distinguishing the maturity levels}

The AI management framework with five maturity levels presents new insights into the evolution of AI activities in companies. In this respect, it is essential to highlight the major differences among the maturity levels. A key difference between Level 0 and Level 1 is activating the relevant persons to enable experimentation with AI technologies. With regard to Level 2, major emphasis is placed on arranging an AI initiative that includes the different AI activities. On this basis, 
firms focus on accomplishing the key tasks to ensure a successful AI execution, which constitutes a core part of Level 3. With respect to Level 4, companies focus their AI initiatives on advancing towards growth and innovation beyond improvements and optimization. Finally, companies concentrate on amalgamating AI and human intelligence at Level 5 of Integrated Intelligence. Only this integration of multiple types of intelligence will enable a sustainable competitive advantage in an intelligence-driven future business context (Lichtenthaler, 2020b).

In this regard, the AI management framework guides future discussions about firms' maturity levels in managing $\mathrm{AI}$ and in achieving competitive benefits at the interfaces of AI and human expertise (Hirsch, 2018; Oliveira, 2017). On this basis, the framework provides an important starting point for standardized AI maturity assessments, which may be particularly important for measuring the dynamic transformations of AI management over time (Wein, 2018; Woyke, 2017). In addition, the framework contributes to explaining many firms' challenges in creating value with AI and in capturing the value of their AI initiatives in terms of an enhanced competitive position (Fountaine, McCarthy, \& Saleh, 2019; Pinto-dos-Santos et al., 2018). For example, if a company remains at Level 2, any AI program is unlikely to produce innovative results that go substantially beyond the optimization of existing business processes. In addition, the five maturity levels help to systematically identify the limitations of AI management even in those companies that are considered to be among the leading firms in managing and profiting from AI (Berman, 2012; Lewrick, Link, \& Leifer, 2018).

\section{Conclusion and outlook}

Based on distinguishing various maturity levels, the AI management framework points to white spots and unrealized opportunities in nearly all companies at present. By addressing these additional opportunities of leveraging AI and achieving an integrated intelligence architecture, firms may overcome the paradoxical situation of being considered AI leaders although they have only captured the low-hanging fruits. In contrast, the major benefits in terms of innovation and competitive advantage have usually not been addressed yet (Davenport \& Ronanki, 2018). This situation of perceived high performance levels may sometimes be even more challenging than the situation of firms that have completely ignored AI activities. In fact, the executives in many firms with AI activities believe that these activities are successful in absolute terms and also relative to their firm's potential (Lichtenthaler, 2020b). Consequently, they are convinced that these activities should be continued in their present form or only need to be slightly adapted and extended.

However, this strategic emphasis usually limits the extent of AI activities to realizing efficiency benefits, whereas most further opportunities in terms of innovation and new business models are left unconsidered (Warner \& Wäger, 2019; Westerman \& Bonnet, 2015). Drawing on an intelligence-based perspective, the core differences among the maturity stages - activating, arranging, accomplishing, advancing, and amalgamating - offer immediate starting points for taking a firm's AI management to the next level based on customized action plans. In addition, they underscore the relevance of an intelligence-based approach to firm performance (Lichtenthaler, 2019). In light of potential future progress in AI technology, firms may not only advance from 
Isolated Ignorance to Integrated Intelligence. Instead, some degree of Intuitive Ingenuity may further expand the strategy space and the competitive alternatives against the background of growing maturity levels of managing AI.

\section{References}

Agrawal, A., Gans, J., \& Goldfarb, A. (2018). Prediction machines: The simple economics of artificial intelligence. Boston: Harvard Business Review Press.

Agrawal, A. K., Gans, J. S., \& Goldfarb, A. (2017). What to expect from artificial intelligence. MIT Sloan Management Review, 58(3): 23-26.

Apanesevic, T., Arvidsson, N., \& Markendahl, J. (2018). Mobile payments: a proposal for a context-oriented approach based on socio-technical system theory. Journal of Innovation Management, 6(3): 40-97.

Berman, S. J. (2012). Digital transformation: opportunities to create new business models. Strategy \& Leadership, 40(2): 16-24.

Brito, C. (2018). Promoting the creation of Innovation Ecosystems: the case of the University of Porto. Journal of Innovation Management, 6(3): 8-16.

Brock, J.K.U., \& Wangenheim, F. von. (2019). Demystifying AI: What Digital Transformation Leaders Can Teach You about Realistic Artificial Intelligence. California Management Review, 61(4): 110-134.

Bühring, J., \& Moore, P. A. (2018). Emotional and Social Intelligence as 'Magic Key' in Innovation: A Designer's call toward inclusivity for all. Journal of Innovation Management, 6(2): $6-12$.

Datta, S.P.A. (2018). Unleashing the new Wealth Of Nations. Journal of Innovation Management, 6(2): 13-37.

Daugherty, P. R., \& Wilson, H. J. (2018). Human + machine: Reimagining work in the age of AI. Boston: Harvard Business Review Press.

Davenport, T. H. (2018). AI Advantage: How to Put the Artificial Intelligence Revolution to Work. Cambridge: MIT Press.

Davenport, T. H., \& Ronanki, R. (2018). Artificial Intelligence for the Real World. Harvard Business Review, 96: 108-116.

Finlay, S. (2017). Artificial Intelligence and Machine Learning for Business: A No-Nonsense Guide to Data Driven Technologies. Preston: Relativistic.

Fountaine, T., McCarthy, B., \& Saleh, T. (2019). Building the AI-Powered Organization. Harvard Business Review, 97(4): 62-73.

Garbuio, M., \& Lin, N. (2019). Artificial Intelligence as a Growth Engine for Health Care Startups: Emerging Business Models. California Management Review, 61(4): 59-83. 
Gartner. (2019a). Artificial intelligence. Information technology glossary:

https://www.gartner.com/en/information-technology/glossary/artificial-intelligence.

Gartner. (2019b). Data management. Information technology glossary:

https://www.gartner.com/en/information-technology/glossary/dmi-data-management-and-integration.

Gartner. (2019c). Enterprise information architecture. Information technology glossary:

https://www.gartner.com/en/information-technology/glossary/enterprise-information-architecture.

Gartner. (2019d). Information architecture. Information technology glossary:

https://www.gartner.com/en/information-technology/glossary/information-architecture.

General Electric. (2019). https://www.ge.com/research/technology-domains/artificial-intelligence.

Haenlein, M., \& Kaplan, A. (2019). A Brief History of Artificial Intelligence: On the Past, Present, and Future of Artificial Intelligence. California Management Review, 61(4): 5-14.

Hirsch, P. B. (2018). Tie me to the mast: Artificial intelligence \& reputation risk management. Journal of Business Strategy, 39(1): 61-64.

Huang, M. H., \& Rust, R. T. (2018). Artificial intelligence in service. Journal of Service Research, 21(2): $155-172$.

Ili, S., \& Lichtenthaler, U. (2017). FAQ Digital Transformation and Artificial Intelligence: 101 Questions - 101 Answers. Karlsruhe: Ili Consulting.

Kaplan, A. M., \& Haenlein, M. (2019). Siri, Siri, in My Hand: Who's the Fairest in the Land? On the Interpretations, Illustrations, and Implications of Artificial Intelligence. Business Horizons, 62(1): 15-25.

Kavadias, S., Ladas, K., \& Loch, C. (2016). Artificial Intelligence Is Almost Ready for Business. Harvard Business Review, 94: 91-98.

Kumar, V., Rajan, B., Venkatesan, R., \& Lecinski, J. (2019). Understanding the Role of Artificial Intelligence in Personalized Engagement Marketing. California Management Review, 61(4): $135-155$.

Lewrick, M., Link, P., \& Leifer, L. (2018). The Design Thinking Playbook: Mindful Digital Transformation of Teams, Products, Services, Businesses and Ecosystems. New York: Wiley.

Lichtenthaler, U. (2018). Substitute or synthesis? The interplay between human and artificial intelligence. Research-Technology Management, 61(5): 12-14.

Lichtenthaler, U. (2019). An intelligence-based view of firm performance: Profiting from artificial intelligence. Journal of Innovation Management, 7(1).

Lichtenthaler, U. (2020a). Beyond artificial intelligence: why companies need to go the extra step. Journal of Business Strategy, 41(1): 19-26.

Lichtenthaler, U. (2020b). Integrated intelligence: Combining human and artificial intelligence for competitive advantage. Frankfurt: Campus. 
Lloyd-Jones, T. (2016). https://blogs.lexisnexis.com/insurance-insights/2016/06/machine-learningartificial-intelligence-insurance/.

Lohr, S., (2004, March 1). Microsoft, Amid Dwindling Interest, Talks Up Computing as a Career. New York Times. Accessed January 2020.

https://www.nytimes.com/2004/03/01/business/microsoft-amid-dwindling-interest-talks-up-computingas-a-career.html.

Metcalf, L., Askay, D. A., \& Rosenberg, L. B. (2019). Keeping Humans in the Loop: Pooling Knowledge through Artificial Swarm Intelligence to Improve Business Decision Making. California Management Review, 61(4): 84-109.

Mueller, J. P., \& Massaron, L. (2018). Artificial Intelligence for Dummies. Hoboken: Wiley.

Oliveira, E. (2017). Beneficial AI: The next battlefield. Journal of Innovation Management, 5(4): $6-17$.

Overgoor, G., Chica, M., Rand, W., \& Weishampel, A. (2019). Letting the Computers Take Over: Using AI to Solve Marketing Problems. California Management Review, 61(4): 156-185.

Pinto-dos-Santos, D., Giese, D., Brodehl, S., Chon, S. H., Staab, W., Kleinert, R., Maintz, D., \& Baessler, B. 2018. Medical students' attitude towards artificial intelligence: a multicentre survey. European Radiology, 1(1): 1-7.

Plastino, E., \& Purdy, M. 2018. Game changing value from Artificial Intelligence: eight strategies. Strategy \& Leadership, 46: 16-22.

Rometty, G. 2016. Digital today, cognitive tomorrow. MIT Sloan Management Review, 58(1): $168-171$.

SAE International. 2019. https://www.sae.org/news/2019/01/sae-updates-j3016-automateddriving-graphic.

Shrestha, Y. R., Ben-Menahem, S. M., \& Krogh, G. von. 2019. Organizational DecisionMaking Structures in the Age of Artificial Intelligence. California Management Review, 61(4): $66-83$.

Swift, T. 2018. PhD scientists in the boardroom: the innovation impact. Journal of Strategy and Management, 11(2): 184-202.

Tambe, P., Cappelli, P., \& Yabukovich, V. 2019. Artificial Intelligence in Human Resources Management: Challenges and a Path Forward. California Management Review, 61(4): 15-42.

Tegmark, M. 2018. Life 3.0: Being human in the age of artificial intelligence. New York: Vintage Books.

Warner, K.S.R., \& Wäger, M. 2019. Building dynamic capabilities for digital transformation: An ongoing process of strategic renewal. Long Range Planning, 52(3): 326-349.

Wein, E. 2018. Artificial Intelligence Making Machines Learn: A friendly Introduction.

Westerman, G., \& Bonnet, D. 2015. Revamping your business through digital transformation. MIT Sloan Management Review, 56: 10-13. 
Wilbert, J.K.W., Durst, S., Ferenhof, H. A., \& Selig, P. M. 2018. Unlearning at the Individual Level: An Exploratory Case Study in a High Power Distance Country. Journal of Innovation Management, 6(3): 17-39.

Wilson, H. J., Daugherty, P., \& Bianzino, N. 2017. The jobs that artificial intelligence will create. MIT Sloan Management Review, 58(4): 14-16.

Wilson, H. J., \& Daugherty, P. R. 2018. Collaborative intelligence: Humans and AI are joining forces. Harvard Business Review, 96(4): 114-123.

Woyke, E. 2017. General Electric builds an AI workforce. MIT Technology Review, Online: $1-3$. 


\section{Biographies}

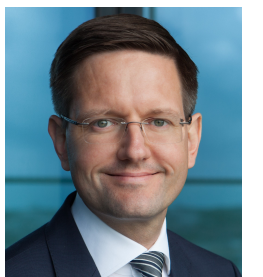

Ulrich Lichtenthaler. Ulrich Lichtenthaler is a Professor of Management and Entrepreneurship at ISM - International School of Management in Cologne, Germany. He holds a Ph.D. degree in technology management and further is an Executive Consultant, who has successfully completed over 20 digital transformation projects over the past years. He has written multiple books and articles for journals and newspapers, such as MIT Sloan Management Review and Wall Street Journal, and he has taught executive education courses at leading business schools. 